\title{
A Multi-Modal Utility to Assist Powered Mobility Device Navigation Tasks
}

\author{
James Poon ${ }^{1}$ and Jaime Valls Miro ${ }^{2}$ \\ 1 University of Technology Sydney, Aus \\ james.poon@student.uts.edu.au \\ 2 University of Technology Sydney, Aus \\ jaime.vallsmiro@uts.edu.au
}

\begin{abstract}
This paper presents the development of a shared control system for power mobility device users of varying capability in order to reduce carer oversight in navigation. Weighting of a user's joystick input against a short-tem trajectory prediction and obstacle avoidance algorithm is conducted by taking into consideration proximity to obstacles and smoothness of user driving, resulting in capable users rewarded greater levels of manual control for undertaking maneuvres that can be considered more challenging. An additional optional comparison with a Vector Field Histogram applied to leader-tracking provides further activities, such as completely autonomous following and a task for the user to follow a leading entity. Indoor tests carried out on university campus demonstrate the viability of this work, with future trials at a care home for the disabled intended to show the system functioning in one of its intended settings.
\end{abstract}

Keywords: shared control, co-autonomy, wheelchair

\section{Introduction}

Powered mobility devices (PMD) including wheelchairs and scooters are quite widely used by aged and disabled people. The use of these aids becomes quite frequent after the age of 65 , with nearly 4.5 million powered wheelchair users in the United States alone [1]. Combined with predictions indicating the global population of people aged over 60 is set to double between 2000 and 2050 [2], there is a drive to improve the methods through which services towards aged and disabled care is delivered. In order to fulfil this need, shared autonomy has become a well-explored research area for assisting PMD users in everyday mobility tasks [3-5]. The goal of shared autonomy is to mitigate the detrimental impact of poor vision or cognitive and physical deficiencies on PMD proficiency through environment-sensing and decision-making rather than relying purely on potentially dangerous user commands. Additionally, novel methods of communicating intent to the PMD including force-feedback modulation of a controller's available range of motion [6] as well as more advanced approaches such as gazetracking [7] and EEG headsets, although the latter may still present some difficulties to cognitively impaired users in cluttered living spaces [8]. These new 
tools and platforms towards enabling disabled individuals to safely undertake everyday mobility tasks independently have the simultaneous effects of reducing the effort on their part, while also helping to mitigate the burden on carers by decreasing the level of supervision required for mobility oversight.

The outcomes of this work were primarily designed around the needs of Greystanes Disability Services (GDS); a care home supporting people with disabilities and complex health needs, with an aim of freeing up staff-hours better utilised in other aspects of patient care. As a carer is required to work with individual patients in everyday mobility activities, shared autonomy could provide a substantially less supervision-intensive alternative where one carer can oversee multiple PMDs simultaneously. Throughout the care home, a shared control mechanism for everyday mobility tasks will also be beneficial to prevent collisions with people, other PMDs or structure while encouraging independent driving on behalf of the user. Additionally, a regular activity is taking multiple wheelchair-bound patients on outdoor excursions through the surrounding bushland environment; currently a rather staff-intensive activity as one staff member is required per wheelchair for the duration of the exercise. It is hence desirable to have a system capable of encouraging patients capable of driving to follow the wheelchair ahead of them or the staff member leading the convoy. The remainder of this paper is structured as follows: Section 2 outlines the test platform and software configuration, Sections 3-4 outline the modes of operation developed, Sections 5-6 document and discuss experimental results, and Section 7 closes with concluding remarks.

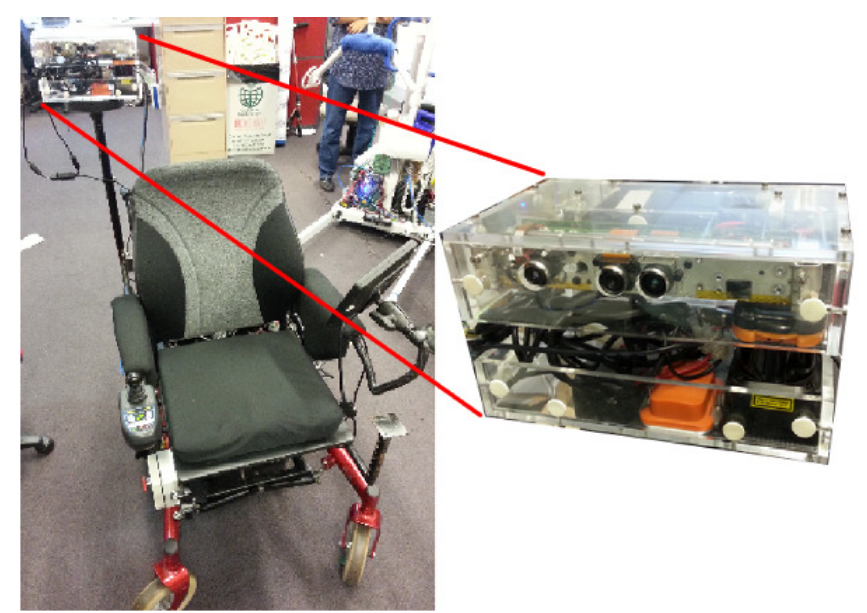

Fig. 1. Instrumented wheelchair with mounted sensor package 


\section{Experiment Setup}

Three distinct modes of operation have been developed:

- Shared control driving with forward projection

- Fully autonomous leader following, disregarding user input

- A semi-autonomous leader-following 'exercise' with sliding-scale autonomy

At present each mode is a standalone item, designed to meet separate healthcare mobility needs. The first encourages disabled users to drive independently, and the second allows carers to move PMD users in cases where driving is prohibitive. The third mode is designed as an engaging activity for users partially capable of driving to safely follow a leader from one location to another; useful in places such as within GDS where groups of people are often moved between areas, for example between a recreational area and a sauna room where several users may wish to drive themselves, but punctuality may be of some priority to carers.

Development and experimentation was conducted on the Centre of Autonomous Systems instrumented wheelchair platform (Fig 1), fitted with drive motors and wheel encoders. An additional sensor module was added, containing a MS Kinect RGB-D camera, Hokuyo laser scanner and an Xsens inertial measurement unit. All sensors and the motor controller interface with the on-board Fit-PC, running Ubuntu 10.04 and utilizing the ROS (Robot Operating System - www.ros.org) middleware. Regardless of the mode of operation selected, at all times a collision prevention safeguard layer sits above the platform driver. Additionally, odometry pose information is combined with laser scanner data to hold environmental information beyond the scanner's field of view.

\section{Shared Control Navigation}

\subsection{Local Planning}
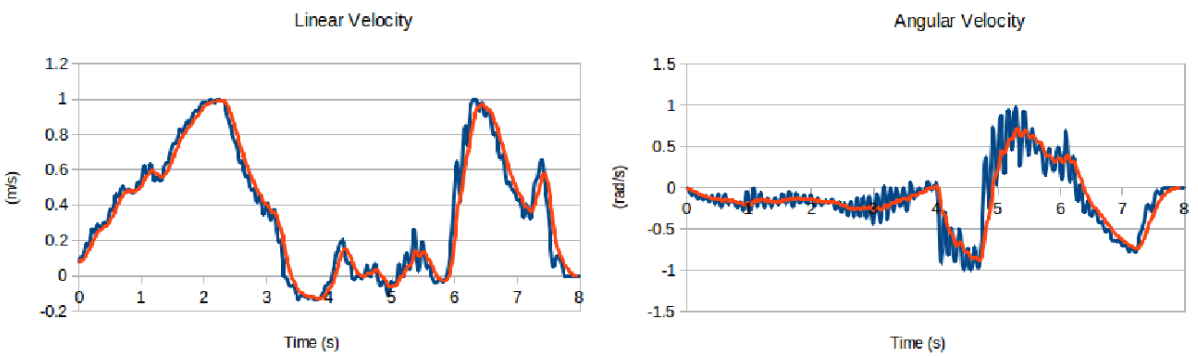

Fig. 2. Weighted average filter (blue to red) 


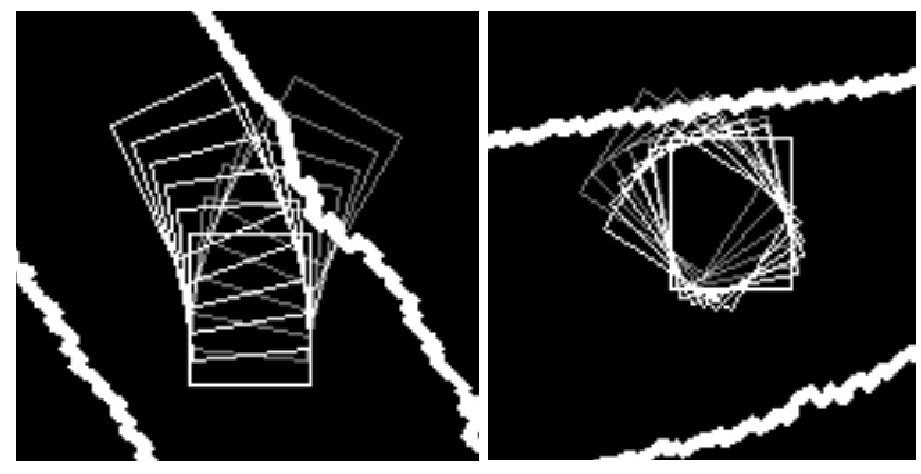

Fig. 3. Trajectory modulation of platform footprint pose from input (grey) to safe (white) when driving and turning in a corridor, indicated by the thicker white lines

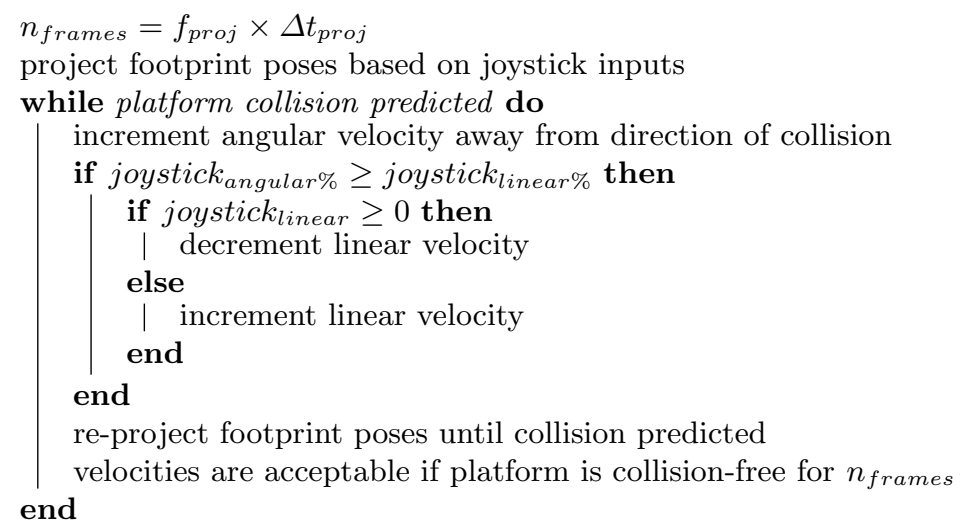

Algorithm 1: Forward-projection pseudocode

As users of PMDs may suffer from involuntary movements such as jerkiness or tremoring [9] it becomes necessary to filter out inputs which may not be indicative of desired platform behaviour. Noise reduction can be done through a multitude of signal processing methods such as weighted average [10] or Kalman filters [11]. There also exist advanced mechanisms for screening out of involuntary yet seemingly fluent input actions through learning frameworks [12], however for the scope of this work filtering was carried out through more conventional noise mitigation approaches under the assumption of tremor suppression. Figure 2 demonstrates the output of a weighted moving-average filter showing significant noise reduction at the cost of a slight time delay. These filtered input velocities are then applied to the PMD's footprint for a brief forward-projection (Algorithm 1), as shown in Figure 3. This allows for responsive local control without prior map-building, enabling functionality in environments often subject to frequent change and/or have many other moving entities, such as a shopping area. 


\section{Leader Following}

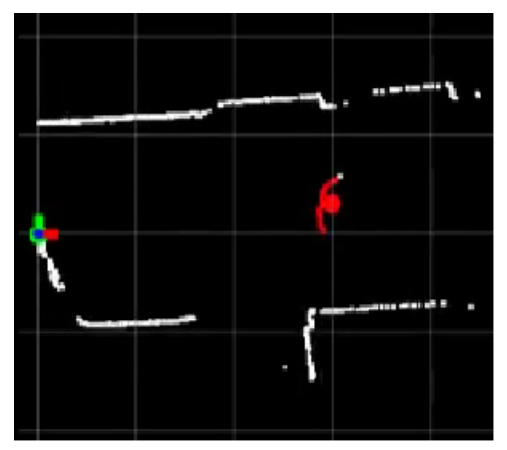

Fig. 4. Tracking (red contour with corresponding dot) using laser scanner

A blob-tracking algorithm was developed to be used with the laser scanner readings. After seeding with an initial pose, a cluster of closely spaced points can be tracked through sequential scans. 'Merging' with other objects in the environment such as walls is mitigated by a maximum search radius heuristic. Figure 4 shows a sample frame from the tracking with the leader highlighted in red. These poses are directly fed as inputs into a Vector Field Histogram [13] (VFH), chosen for its suitability in planning to local goals. A VFH determines safe control speeds based on a polar obstacle grid of the platform's immediate surroundings. In the case of autonomous following, the resultant velocity commands are fed directly to the safeguard layer. For the following exercise, the user weight $\eta$ (Section 4.1) is applied between the VFH output $v, w_{v f h}$ and the user's filtered input $v, w_{\text {filter }}$ (Eqns 1-2). Full autonomy is enabled if the user releases the joystick, in order to avoid interruptions to the platform's movement which the leader may not be able to notice immediately.

$$
\begin{aligned}
& v= \begin{cases}v_{v f h} & \text { if } v_{f i l t e r}=\omega_{f i l t e r}=0 \\
\eta \times v_{\text {filter }}+(1-\eta) \times v_{v f h} & \text { otherwise }\end{cases} \\
& \omega= \begin{cases}\omega_{v f h} & \text { if } v_{\text {filter }}=\omega_{\text {filter }}=0 \\
\eta \times \omega_{\text {filter }}+(1-\eta) \times \omega_{v f h} & \text { otherwise }\end{cases}
\end{aligned}
$$

\subsection{Weighting}

$$
\begin{aligned}
\eta_{s a} & =1-e^{-\alpha \times d_{\min }} \\
\eta_{s m} & =e^{-\beta \times \delta_{a x i s}} \\
\eta_{o b} & =e^{-\gamma \times\left|\omega_{\text {track }}-\omega_{\text {filter }}\right|}
\end{aligned}
$$




$$
\begin{aligned}
& \eta=\min \left(\eta_{s a}, \eta_{s m}, \eta_{o b}\right) \\
& \eta= \begin{cases}0 & \text { if } \eta \leq \eta_{0} \\
1 & \text { if } \eta \geq \eta_{1}\end{cases}
\end{aligned}
$$

The user weight is primarily determined using two or three proficiency metrics $[14,15]$ of safety (Eqn 3), smoothness (Eqn 4) and 'obedience' (Eqn 5). $d_{\min }$ represents the closest obstacle to the platform footprint and $\delta_{\text {axis }}$ represents a percentage change in joystick axis position per second. Obedience is determined by the difference between the angular velocities of the VFH and the filtered joystick input, only taken into account when leader tracking is active. The gains $\alpha, \beta$ and $\gamma$ were roughly determined from desired values of $\eta$ at specific values; for instance $\beta$ was chosen to be 0.0035 from a desired $\eta_{s m}$ of 0.5 at $200 \%$ axis movement per second. A higher weighting gives the user a greater level of permitted deviation from what is considered a safe local trajectory to permit closer proximity to hazards, and a lower weighting tends to limit the user to a more conservative style of driving. Weighting (Eqns 6-7) is then used to blend filtered input velocities with the outputs from the local planner (Eqns 8-9). In our experiments, cutoff values for $\eta_{0}$ and $\eta_{1}$ were 0.25 and 0.75 respectively.

$$
\begin{aligned}
v & =\eta \times v_{\text {filter }}+(1-\eta) \times v_{\text {planner }} \\
\omega & =\eta \times \omega_{\text {filter }}+(1-\eta) \times \omega_{\text {planner }}
\end{aligned}
$$

\section{Results}

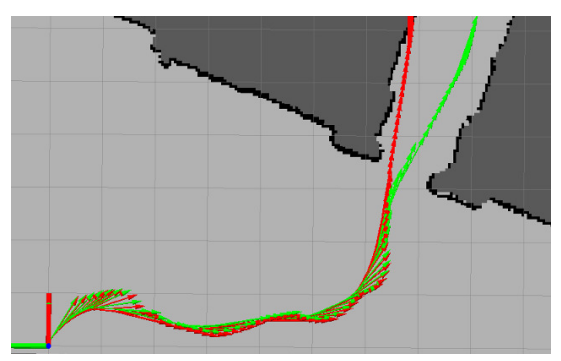

Fig. 5. Assisted driving through narrow doorway (green) compared to raw input (red)

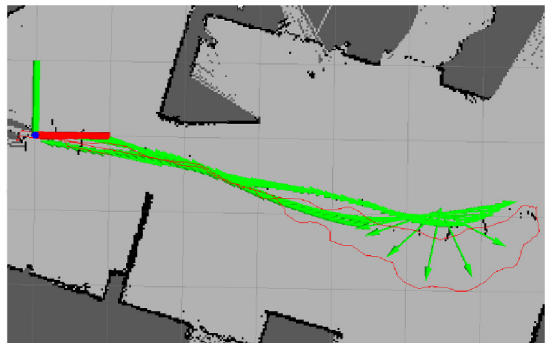

Fig. 6. Autonomous following (green) through doorway via VFH behind leader entity (red)

Preliminary tests were conducted on the University of Technology, Sydney campus. As navigating areas such as doorways and narrow hallways presents 


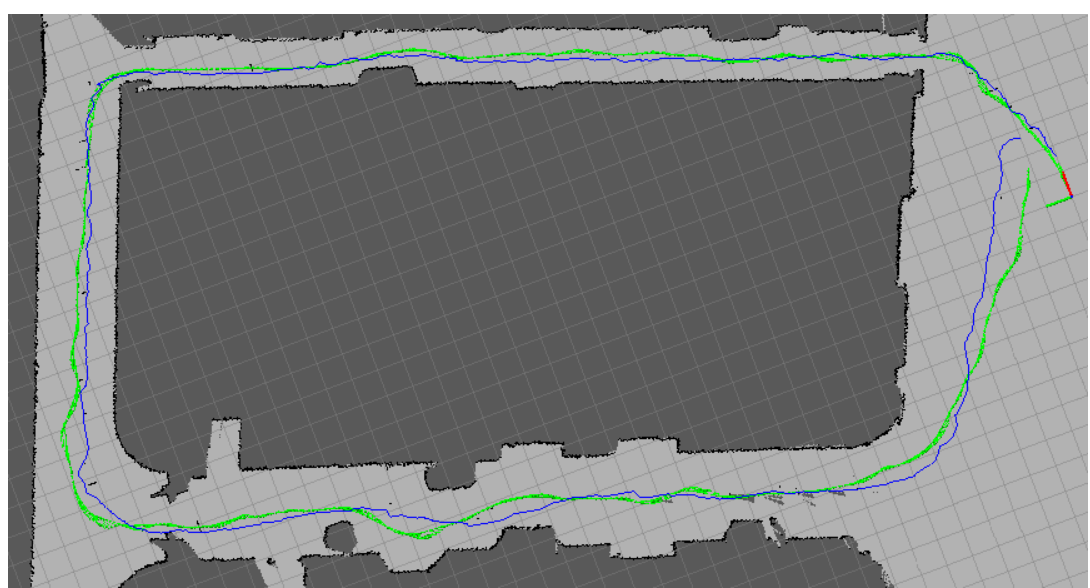

Fig. 7. Leader (blue) following exercise in counter-clockwise indoor loop

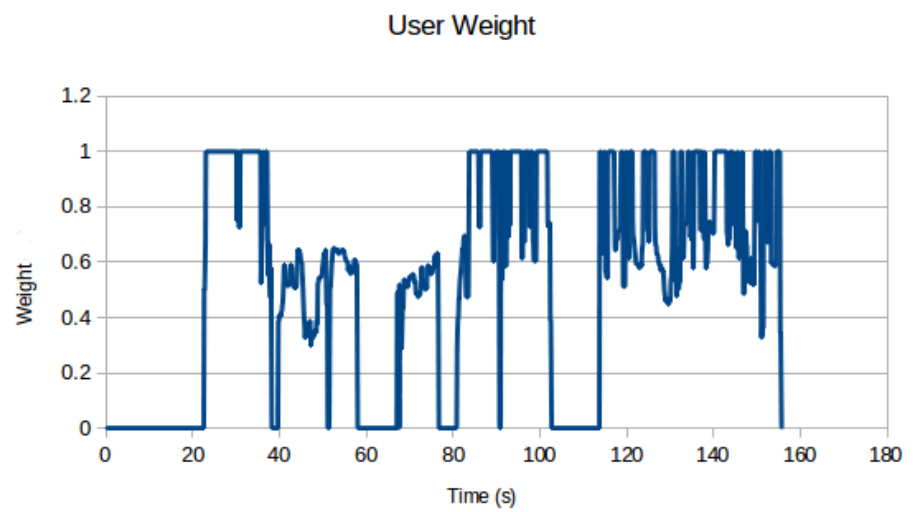

Fig. 8. User weighting results for following exercise

particular difficulty [16] to the average PMD user, experimentation focused on suitably constrained spaces such as those that may be found in a 'normal' interior space not necessarily configured to accomodate PMDs. Figure 5 shows the trajectory difference in trajectories between the joystick input (red) and the output of the shared control algorithm (green) when roughly aimed at a doorway with $\sim 5 \mathrm{~cm}$ clearance on either side. Figure 6 shows the trajectories from the VFH (green) against the leader's path from laser scanner tracking (red) through another doorway with $\sim 10 \mathrm{~cm}$ clearance twice, with a tight on-the-spot turn in between. Trajectories and maps were produced via Hector mapping [17]; map regions are as follows: light grey represents known vacant space, black represents known surfaces and dark grey represents unknown space; the grid cells are 1 metre square. 
Figure 7 shows the trajectory taken by the wheelchair (green) when the user attempts to follow a leader (blue). Figure 8 shows the corresponding control weight allocated to the user. When driving is erratic or tight spaces are encountered, the weighting is reduced to allow mediation from the VFH in order to stay truer to the leader trajectory or to safely bypass the potential hazard. The test user also allowed the VFH to fully take over momentarily in a few areas by releasing the joystick, resulting in the lengthier $0 \%$ weighting periods visible.

\section{Discussion}

Despite a major drawback of these results being that the manual driving components were conducted by an able-bodied user attempting to emulate a PMD user unable to smoothly or confidently maneuvre through tight spaces, the early results appear to positively address the project's needs. The modes of operation evaluated in these experiments all feature distinct value in healthcare, from enabling patients to safely move themselves or a single staff to convoy several PMDs, to a mobility exercise providing engagement to users while guiding them to their destination. The benefits of the latter extend beyond its intrinsic value by additionally providing a potential means of PMD training or basic proficiency assessment. Providing users accustomed to staff supervision with the opportunity to use intelligent PMDs unsupervised may likely require some training and initial acclimation, however the benefits to the patients' self-esteem from maintained independence and to staff by reducing time spent guiding PMD users would be considerable.

Testing these solutions at Greystanes Disability Services with disabled patients is presently of high priority to assess the system's performance in real test cases, as well as to obtain feedback and suggestions from the system's intended users when comparing the system's outputs to raw user driving. The development of a simple GUI to handle the execution of relevant softwares for each mode would be highly beneficial to staff and some PMD users, and to unify these solutions into a single utility. Other future planned developments include a more sophisticated noise mitigation algorithm for involuntary movements that may not be simple tremors, and an improved planner for more complex local trajectories beyond basic forward projection. Different sensors may be required to detect hazards on the ground or objects below the 2D laser scanner's height such as furniture items or household utilities. Obstacles on the ground may not be a significant concern indoors, however as outdoor use is intended a smooth traversable surface cannot always be ensured. Outdoor applications must also take into account obstacles identified by PMD users to be difficult to pass [18] such as groups of people. Downward-pitched stereo cameras or an IR depth imager could be used to provide height-maps and additional stability/traversability information $[19,20]$ for planning algorithms; a study left for future development and investigation. 


\section{Conclusions}

This paper describes several solutions to assist caregiver staff by reducing the need to monitor PMD users in everyday mobility tasks related to patient care. Each approach has been demonstrated to be capable of providing navigational support across several different scenarios including safeguarded driving, autonomous following and a hybrid driving exercise. The reduced requirement for manual oversight would provide staff with more time towards less rudimentary tasks, while additionally enhancing the self-esteem and personal independence of disabled PMD users. Despite the shortcomings of the experiments conducted we believe the outcomes are relevant in the context of this work, and hope to follow these developments with a larger trial at a care home involving several disabled users under adequate supervision from qualified care staff.

Acknowledgements: The authors would like to thank Greystanes Disability Services - http://www.greystanes.org.au) for their collaboration with this research project.

\section{References}

1. Kaye, H.S. and Kang, T. and LaPlante, M.P.: Mobility Device Use in the United States. Disability Statistics Report 14. Disability Statistics Center, University of California, San Francisco. (2000)

2. Congressional Budget Office (C.B.O.): Global population aging in the 21st century and its economic implications. (2005) http://www.cbo.gov/sites/default/files/cbofiles/ftpdocs/69xx/doc6952/ 12-12-global.pdf. Accessed: May 14, 2014.

3. Lankenau, A. and Meyer, O. and Krieg-Bruckner, B.: Safety in robotics: the Bremen Autonomous Wheelchair. Advanced Motion Control, 1998 5th International Workshop on. 524-529 (1998)

4. Pires, G. and Honorio, N. and Lopes, C. and Nunes, U. and Almeida, A.T.: Autonomous wheelchair for disabled people. Industrial Electronics, Proceedings of the IEEE International Symposium on. 3, 797-801 (1997)

5. Oishi, M. and Cheng, A. and Bibalan, PT. and Mitchell, I.: Building a smart wheelchair on a flexible software platform. RESNA International Conference on Technology and Aging. (2011)

6. Masone, C. and Franchi, A. and Bulthoff, H.H. and Giordano, P.R.: Interactive planning of persistent trajectories for human-assisted navigation of mobile robots. Intelligent Robots and Systems (IROS), 2012 IEEE/RSJ International Conference on. 2641-2648 (2012)

7. Al-Haddad, A. and Sudirman, R. and Omar, C.: Gaze at Desired Destination, and Wheelchair Will Navigate towards It. New Technique to Guide Wheelchair Motion Based on EOG Signals. Informatics and Computational Intelligence (ICI), First International Conference on. 126-131 (2011)

8. Graham-Rowe, D.: Wheelchair makes the most of brain control. (2010) http://www.technologyreview.com/news/420756/ wheelchair-makes-the-most-of-brain-control/. Accessed: Apr 3, 2014. 
9. van der Zwaag, B.J. and Corbett, D. and Jain, L.: Minimising tremor in a joystick controller using fuzzy logic. Knowledge-Based Intelligent Information Engineering Systems, 1999. Third International Conference, 5-8 (1999)

10. Bing-Yuh Lu and Chen-Yuan Liu and JerJunn Luh and Chao-Chih Shiao and Fok Ching Chong: Standard Deviation Based Weighted Average Function for Reducing Tremor Disturbance of the Joystick. e-Health Networking, Application and Services, 2007 9th International Conference on. 214-216 (2007)

11. Welch, G. and Bishop, G.: An Introduction to the Kalman Filter (2006) http://www.cs.unc.edu/ welch/media/pdf/kalman_intro.pdf. Accessed: Apr 22, 2014.

12. Demeester, E. and Nuttin, M. and Vanhooydonck, D. and Van Brussel, H.: A model-based, probabilistic framework for plan recognition in shared wheelchair control: experiments and evaluation. Intelligent Robots and Systems, 2003. (IROS 2003). Proceedings. 2003 IEEE/RSJ International Conference on, 2:1456-1461 (2003)

13. Borenstein, J. and Koren, Y.: The vector field histogram-fast obstacle avoidance for mobile robots. Robotics and Automation, IEEE Transactions on. 7(3):278-288 (1991)

14. Qinan Li, Weidong Chen, and Jingchuan Wang: Dynamic shared control for humanwheelchair cooperation. Robotics and Automation (ICRA), 2011 IEEE International Conference on. 4278-4283 (2011)

15. C. Urdiales, J.M. Peula, M. Fdez-Carmona, C. Barru, E.J. Prez, I. Snchez-Tato, J.C. Toro, F. Galluppi, U. Corts, R. Annichiaricco, C. Caltagirone, and F. Sandoval: A new multi-criteria optimization strategy for shared control in wheelchair assisted navigation. Autonomous Robots. 30(2):179197 (2011)

16. Koontz, A.M. et al.: Design Features That Affect Maneuverability of Wheelchairs and Scooters. Archives of Physical Medicine and Rehabilitation. 91(5):759-764 (2010)

17. S. Kohlbrecher and J. Meyer and O. von Stryk and U. Klingauf Kolbrecher, S. and Meyer, J. and von Stryk, O. and Klingauf, U.: A Flexible and Scalable SLAM System with Full 3D Motion Estimation. Proc. IEEE International Symposium on Safety, Security and Rescue Robotics (SSRR). (2011)

18. Torkia, C. et al.: Power wheelchair driving challenges in the community: a users perspective. Disabil Rehabil Assist Technol. (2014)

19. Murarka, A. and Kuipers, B.: A stereo vision based mapping algorithm for detecting inclines, drop-offs, and obstacles for safe local navigation. Intelligent Robots and Systems, 2009 (IROS 2009). IEEE/RSJ International Conference on. 16461653 (2009)

20. Norouzi, M. and Miro, J.V. and Dissanayake, G.: A statistical approach for uncertain stability analysis of mobile robots. Robotics and Automation (ICRA), 2013 IEEE International Conference on. 191-196 (2013) 